\title{
Improving feeding and growth of HIV- positive children through nutrition training of frontline health workers in Tanga, Tanzania
}

Bruno F. Sunguya ${ }^{1 *}$, Linda B. Mlunde ${ }^{2}$, David P. Urassa ${ }^{1}$, Krishna C. Poudel ${ }^{3}$, Omary S. Ubuguyu ${ }^{4}$, Namala P. Mkopi ${ }^{4}$, Germana H. Leyna', Anna T. Kessy ${ }^{1}$, Keiko Nanishi ${ }^{2}$, Akira Shibanuma², Junko Yasuoka ${ }^{2}$ and Masamine Jimba ${ }^{2}$

\begin{abstract}
Background: Nutrition training can boost competence of health workers to improve children's feeding practices. In this way, child undernutrition can be ameliorated in general populations. However, evidence is lacking on efficacy of such interventions among Human Immunodeficiency Virus (HIV)-positive children. We aimed to examine the efficacy of a nutrition training intervention to improve midlevel providers' (MLPs) nutrition knowledge and feeding practices and the nutrition statuses of HIV-positive children in Tanga, Tanzania.

Methods: This cluster-randomized controlled trial was conducted in 16 out of 32 care and treatment centers (CTCs) in Tanga. Eight CTCs were assigned to the intervention arm and a total of 16 MLPs received nutrition training and provided nutrition counseling and care to caregivers of HIV-positive children. A total of 776 pairs of HIV-positive children and their caregivers were recruited, of whom 397 were in the intervention arm. Data were analyzed using instrumental variable random effects regression with panel data to examine the efficacy of the intervention on nutrition status through feeding practices.

Results: Mean nutrition knowledge scores were higher post-training compared to pre-training among MLPs (37.1 vs. $23.5, p<0.001$ ). A mean increment weight gain of $300 \mathrm{~g}$ was also observed at follow-up compared to baseline among children of the intervention arm. Feeding frequency and dietary diversity improved following the intervention and a 6 months follow-up $(p<0.001)$. An increase in each unit of feeding frequency and dietary diversity were associated with a 0.15 -unit and a 0.16 -unit respectively decrease in the child underweight $(p<0.001)$.

Conclusions: Nutrition training improved nutrition knowledge among MLPs caring for HIV-positive children attending CTCs in Tanga, Tanzania. Caregivers' feeding practices also improved, which in turn led to a modest weight gain among HIV-positive children. To sustain weight gain, efforts should be made to also improve households' food security and caregivers' education in addition to inservice nutrition trainings. The protocol was registered on 15/02/2013, before the recruitment at ISRCTN trial registry with the trial registration number: ISRCTN65346364.
\end{abstract}

Keywords: Nutrition training, Feeding practices, Nutrition status, HIV/AIDS, Midlevel providers

\footnotetext{
* Correspondence: sunguya@gmail.com

${ }^{1}$ School of Public Health and Social Sciences, Muhimbili University of Health

and Allied Sciences, P.O Box 65489, Dar es Salaam, Tanzania

Full list of author information is available at the end of the article
}

(c) The Author(s). 2017 Open Access This article is distributed under the terms of the Creative Commons Attribution 4.0 International License (http://creativecommons.org/licenses/by/4.0/), which permits unrestricted use, distribution, and reproduction in any medium, provided you give appropriate credit to the original author(s) and the source, provide a link to the Creative Commons license, and indicate if changes were made. The Creative Commons Public Domain Dedication waiver (http://creativecommons.org/publicdomain/zero/1.0/) applies to the data made available in this article, unless otherwise stated. 


\section{Background}

The global burden of child undernutrition is declining. However, the rates still vary widely among low-income countries [1], with the brunt of the burden of undernutrition still falling on just a few [2]. For example, only 14 countries - all low-income - harbor $80 \%$ of the world's stunted children [2]. Poor feeding practices [3], food insecurity [4], and poverty are important factors behind such undernutrition. These countries also suffer from heavy burdens of Human Immunodeficiency Virus/Acquired Immunodeficiency Syndrome HIV/AIDS and, which further worsens child undernutrition [5].

In low-income countries, undernutrition among HIVpositive children is largely associated with poor feeding practices, low education levels among caregivers, and poverty. Food insecurity is persistent among families of HIV-positive children and is also associated with child undernutrition $[4,6]$. However, even in regions with high food production, HIV-positive children are subject to high levels of undernutrition $[7,8]$. In such regions, caregivers with poor nutrition knowledge are more likely to feed their children with a low quality and diversity of foods and at a lower frequency than recommended [7, 9]. Improving nutrition knowledge within such contexts may help to ameliorate child undernutrition.

Caregivers' nutrition knowledge can be improved if they are properly counseled on proper feeding practices based on the local food availability [10]. To achieve this, health workers should first be provided with updated nutrition knowledge, skills, and competence to manage undernutrition. Such skills can be acquired through inservice nutrition training $[11,12]$. Nutrition training of health workers has also been effective to improve feeding practices including feeding frequency, dietary diversity, and dietary adequacy [13]. Thus, nutrition counseling by trained health workers has the potential to improve the growth [14] and livelihood of children in the general population [15].

Although evidence is available among children of general populations, evidence on the efficacy of nutrition training and counseling among HIV-positive children remains lacking despite the higher risk of undernutrition and mortality among them. Moreover, typical inservice nutrition training has mostly involved qualified health workers such as qualified nurses, nutritionists, dieticians, clinicians, and other specialized health workers [12, 16-19]. Just a few nutritiontraining interventions have also included community health workers and non-medical personnel [20-22]. No study, meanwhile, has reported on any nutrition training being provided to midlevel providers (MLPs).

MLPs constitute the majority of health workers in many developing countries, including Tanzania [23]. Because of health workforce shortages [24], MLPs in Tanzania are left to work in rural and semi-urban areas, where a high number of patients also reside [23]. They receive a 2- to 3-year post-secondary school training to care for simple health conditions [23]. Such training may not be adequate to make them competent to treat complex medical conditions such as severe undernutrition of HIV-positive children with other complications. However, they may be the only available workforce to provide such highly demanding care, with minimal support or trainings in some areas. Therefore, this study had two objectives: first to examine the efficacy of MLPs' nutrition training to improve their nutrition knowledge, and second to examine the efficacy of such training to improve caregivers' feeding practices along with the nutrition statuses of affected children.

\section{Methods}

\section{Study design and area}

We conducted this cluster-randomized trial in care and treatment centers (CTCs) in Tanga region, Tanzania. The CTC was taken as the unit of randomization. Detailed information on the CTCs and on the overall health system in this region have been discussed elsewhere $[6,25]$. A total of 16 CTCs which fulfilled the selection criteria out of a total 32 in the study area were randomized into intervention and control arms [25], with a total of 8 assigned to each arm using a coin flip randomization method. Pairs of HIV-positive children and their caregivers who attended the selected CTCs were recruited to participate in this study. Two MLPs were selected from each CTC, and only those of the intervention arm received the inservice nutrition training [10]. All participants and their MLPs were blinded with regard to their allocation status. The protocol was registered in February $15^{\text {th }} 2013$ with a registration number ISRCTN65346364. The recruitment started in July $1^{\text {st }} 2013$ and ended in July $30^{\text {th }} 2014$. The manuscript adhered to the CONSORT guidelines.

Undernutrition is rampart among HIV-positive children attending CTCs in this region. In the formative research study preceded the current cluster-randomized controlled trial, about $62 \%$ of 748 HIV-positive children recruited among those attending the 9 selected CTCs were stunted [6]. About 39\% of them also suffered from low weight for their age. The recruited children had poor feeding frequency and dietary diversity [6]. Reasons proposed for such poor feeding practices included poor caregivers' nutrition knowledge, food insecurity in families of HIV-positive children, and poverty. Such unprecedented magnitudes of undernutrition were higher compared to the situation among children in the general population in the same region. Prevalence of stunting and underweight were 49.4 and $24.1 \%$ respectively among children of the general population in the same region in the year that preceded the current study [26]. 


\section{Participants}

We recruited three groups of participants in this study: HIV-positive children attending HIV CTCs in the Tanga region; caregivers of such children, who accompany them to the CTCs and supervise their medical and nutritional care at home; and the MLPs who provide nutrition care to the HIV-positive children. The inclusion criteria for HIV-positive children included: children aged 6 months to 14 years, registered at the selected CTCs during baseline phase, and accompanied by his/her caregiver. We excluded children whose caregivers refused to participate, those who lacked a confirmatory HIV test, and those without Antiretroviral therapy (ART) information from the medical data.

We defined a child's caregiver as a parent or any other adult providing care for the child, accompanying him/ her to the clinic $[5,27]$, and supervising his/her medical and nutritional care. In the intervention arm, nutrition counseling was provided to such caregivers [13].

We selected MLPs based on their roles in HIV-positive children's routine management and care in their CTCs [25]. We excluded MLPs who did not fit the standard definition [23], including community health workers, homebased caregivers, and other health promotion non-clinical health aides.

Details of sample size calculation were presented in the published research protocol [25]. The minimum calculated sample size was estimated to be 192 pairs of HIVpositive children and their caregivers for each arm (i.e. intervention and control arms). We expanded the sample size to 400 per arm to counteract the effect of loss during follow-up, refusal to continue with the study, children's attendance without caregivers, and missing data.

\section{Intervention and follow-up}

A total of 16 MLPs in the intervention arm received the $13 \mathrm{~h}$ and $40 \mathrm{~min}$ nutrition training conducted for two consecutive days in Korogwe district, Tanga. The training was organized into a total of 18 sessions, based on the standard Guidelines for an Integrated Approach to the Nutritional Care of HIV-infected Children (6 months to 14 years) produced by the World Health Organization (WHO) [10]. The sessions included theory, practice, and role-playing. Practice sessions involved demonstrations and actual clinical management of undernutrition among HIV-positive children and were carried out in a nearby district hospital. Contents of the training were modified to include risk factors pertinent to the HIVpositive child population in Tanga, feeding practices, and available foods as found in formative research [6].

The trained MLPs provided tailored nutrition counseling and management of undernutrition to HIV-positive children and their caregivers attending monthly to their CTCs. This included assessing nutritional needs, making a nutrition care plan, and providing counseling based on the locally available foods and needed amounts thereof. MLPs also assessed children's nutritional statuses, and managed undernutrition and other ailments associated with undernutrition. They followed up on observed improvements or deteriorations in feeding practices, weight, and height.

MLPs of the control arm, meanwhile, continued with their standard care for HIV-positive children [5]. This included clinical HIV-staging, adherence counseling, provision of ART, and management of opportunistic infections, similar to MLPs of the intervention arm.

Both intervention and control arms were followed for a period of six months. During the follow-up, we measured feeding practices, nutrition status, and other healthrelated parameters at the end of the trial and compared them with those observed at baseline.

\section{Measurements}

The outcome variable was nutrition status of HIV-positive children. We intended to measure nutrition status through underweight, wasting, and thinness. To achieve this, we measured weight using the hanging Salter scale ${ }^{\bullet}$ (UK) with minimal clothing for young children. We used Salter digital scale $^{\circledast}$ (Brooklyn, USA) for older children who could stand [28]. We measured height for the 24 months and older children using a Seka ${ }^{\circledR}$ measuring rod [28], and using a marked measuring board in a recumbent position for younger children [29].

We converted the anthropometrics into nutrition indices using the 2006 WHO growth standards [30]. We used the STATA igrowup package to convert measurements into weight-for-age $\mathrm{z}$-scores (WAZ-scores), body mass indexfor-age z-scores (BMIAZ-scores), and weight-for-height zscores (WHZ-scores). WAZ was measured for children aged 6 months to 120 months. In this study, a total of 486 children were in this age group. WHZ-score for children aged 6 months to 60 months, of which, only 160 children were eligible for this measure. On the other hand, BMIAZscores is used to measure thinness for children up to 14 years of age [31, 32]. This means, all children recruited were eligible for this measure. The z-scores were used as continuous variables for all nutrition statuses to capture the trivial changes. WAZ-score below -2 Standard Deviations (SD) was categorized as underweight. Also, WHZ-score below -2SD categorized as wasting and BMIAZ-score below -2SD categorized as thinness.

We measured feeding practices using feeding frequency and dietary diversity scores. Like in previous studies $[5,6$, 27], we asked the caregivers of HIV-positive children about the times they had fed their children in the previous $24 \mathrm{~h}$. We also measured dietary diversity by asking caregivers to provide a list of foods they had fed to their children in the previous $24 \mathrm{~h}$. We made a dietary diversity score based on the list of common foods recalled $[6,25]$. 
We measured nutrition knowledge of MLPs using a standard questionnaire included in the training materials associated with the nutrition training [10]. First, we measured the general knowledge on health- and nutritionrelated aspects using scores of the 40 items in all eight sections of the nutrition-training questionnaire. Second, we measured specific aspects of knowledge as follows: three sections (12 items) on general HIV knowledge; one section (4 items) on food preparation knowledge; two sections (8 items) on child feeding practices knowledge; and two sections (8 items) on nutrition counseling skills knowledge. One point was awarded when a participant responded correctly to the given item and zero points were given for a wrong response. For the general knowledge sections, the total scores ranged from 0 to 40. On specific aspects, scores for general HIV knowledge ranged from 0 to 12. For knowledge on food preparation hygiene, scores ranged from 0 to 4. For feeding practices and counseling skills, total scores ranged from 0 to 8 for each. Details of measurements of wealth index, household food insecurity, ART, and HIV clinical stages are found in a protocol paper [25].

\section{Data collection}

Like in our previous studies in Tanzania [5, 6, 27], we used a pretested Swahili questionnaire that was developed in English, translated into Swahili, and then backtranslated into English by different researchers to ensure retention of meaning for all variables. Trained research assistants, who were also used in the formative research phase [6], received training on the questionnaire contents, ethics, and anthropometric measurement methods. We conducted face-to-face interviews with the caregivers of HIV-positive children, measured children's weight and height, and retrieved medical data from their records [25]. Self-administered questionnaires were used to assess the nutrition knowledge of MLPs in the intervention arm before and after the nutrition training.

\section{Analysis}

We analyzed data using both descriptive methods and regression analyses. For descriptive statistics, we used chisquare and t-tests to compare characteristics of participants in the intervention arm and control arm. The compared variables included demographic characteristics, feeding practices, and nutrition status.

We tested the hypotheses using instrumental variable random effects regression analysis [33]. The analysis used panel data to include only the participants who had data at baseline and final follow-up. This two-stage regression analysis first examined whether the nutrition training improved feeding practices (i.e., feeding frequency or dietary diversity) after adjusting for age, sex, caregiver's education level, household wealth index, and food security.
The second stage of the regression analysis aimed to examine the effect of improved feeding practices. This random effects regressions therefore included either of feeding practices (feeding frequency of dietary diversity) as independent variables and examined changes in nutrition status with two models: underweight and thinness models. The two separate models were built because of the differences between the two outcome variables. In all the models we adjusted for age, sex, education level of the caregiver, household wealth index, and food security. The interaction term of intervention and follow-up was the instrument in this instrument variable random effect model regression. Feeding practices i.e. feeding frequency and dietary diversity was the instrument variable. In this case, feeding frequency or dietary diversity was instrumented by the interaction term of intervention and follow-up. We did not build a wasting-model owing to small number of children with wasting and small sample size of under-five children.

We calculated the effect size of this intervention using Number Needed to Treat (NNT). NNT was calculated while accounting for cluster effects and confounding variables based on the odds ratio (OR) and patient expected event rate (PEER) using the formula from a similar study [34] as follows:

$$
\mathrm{NNT}=[(1-(\mathrm{PEER}(1-\mathrm{OR}))) /(1 \text {-PEER }) \text { PEER }(1-\mathrm{OR})]
$$

OR was calculated using logistic regression models with random effects for clusters and adjusted for age, sex, caregiver's education, wealth index, and food insecurity. PEER was estimated using the event rate of the control group. We used the intention-to-treat principle to analyze the data and set the statistical significance at $p<0.05$. All analyses were conducted using STATA version 12 (StataCorp, College Station, Texas, USA).

\section{Ethical considerations}

We obtained informed written consent from the training participants and caregivers of HIV-positive children before data collection. This study was approved by the Research Ethics Committee of the University of Tokyo, and the Expedited Review Sub-committee of the Senate Research and Publication Directorate of the Muhimbili University of Health and Allied Sciences.

\section{Results}

General characteristics of intervention and control arms Data from 776 pairs of HIV-positive children and their caregivers were available for analysis at baseline. Among them, 397 pairs belonged to the CTCs of the intervention arm (Fig. 1). At the final follow-up, data from 745 pairs were available. Among them, 383 pairs belonged to the intervention arm. 


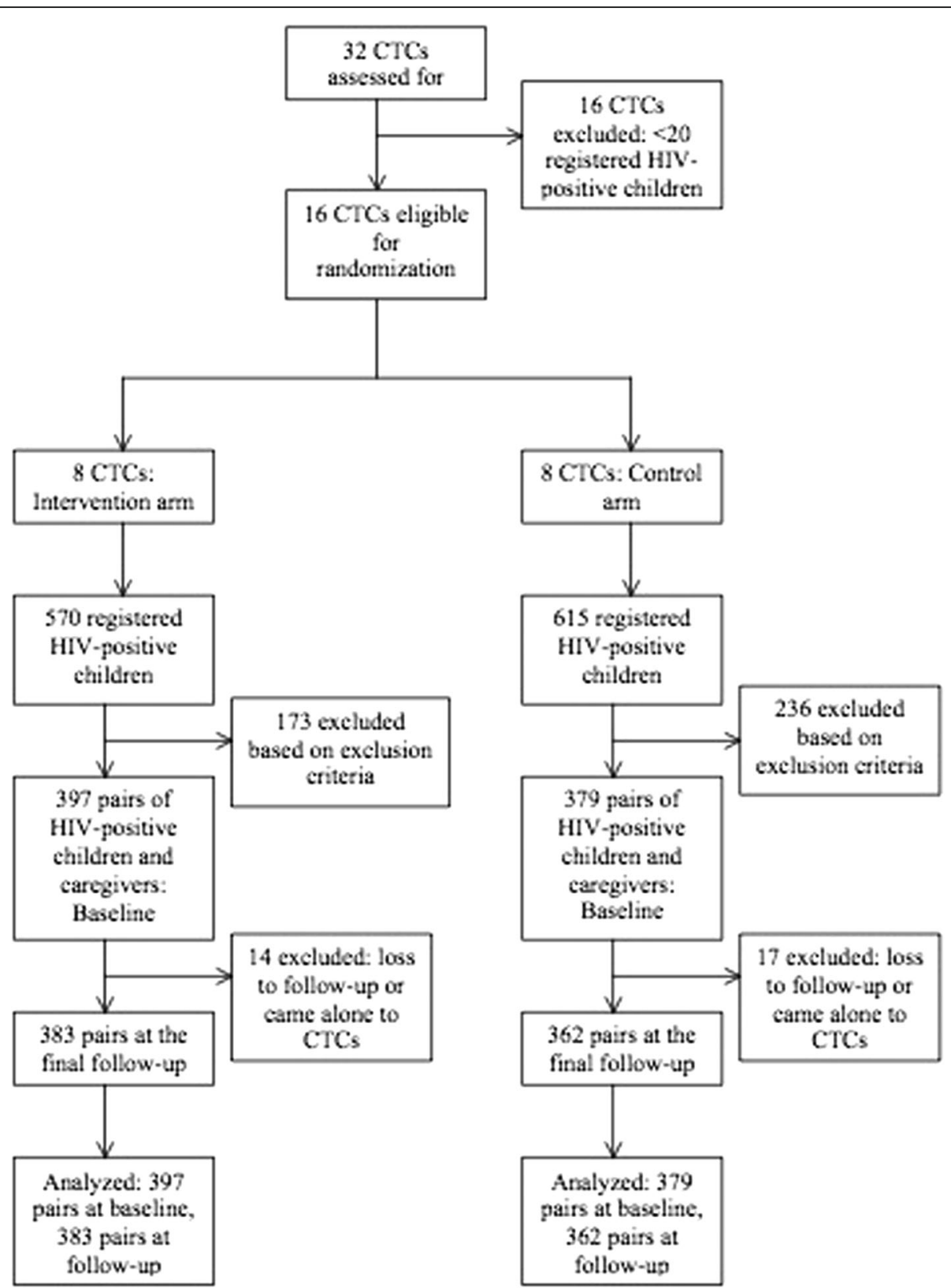

Fig. 1 Trial flow chart

Table 1 shows the general characteristics of participants and compares intervention and control arms. Majority of HIV-positive children $(59.4 \%$ for the intervention and $64.5 \%$ for the control arm) had lost one or both parents. Majority of children in this study were on ART $(86.4 \%$ in the intervention and $88.9 \%$ in the control arm). The mean ART duration for the intervention arm was 36.9 months compared to 33.5 months among those in the control arm. However, $72.8 \%$ of the HIV-positive children in the intervention arm and $72.2 \%$ of those in the control arm had advanced HIV clinical stages. Finally, $68.3 \%$ and $72.6 \%$ of children in the intervention and control arms, respectively, lived in households with food insecurity.

\section{Effectiveness of nutrition training in improving nutrition knowledge}

Table 2 shows the effect of nutrition training on MLPs knowledge, including nutrition knowledge aspects. The knowledge score of MLPs improved after the training compared to the pre-training test (37.1 vs. 23.5, $p<0.001)$. Moreover, all four main aspects of knowledge scores tested in this study improved significantly at the post-training test compared to the 
Table 1 Descriptive characteristics of intervention and control arms

\begin{tabular}{|c|c|c|c|c|c|c|c|c|}
\hline \multirow[t]{2}{*}{ Variable } & \multirow[t]{2}{*}{ Total } & \multicolumn{3}{|c|}{ Intervention } & \multicolumn{3}{|c|}{ Control } & \multirow[t]{2}{*}{$P$} \\
\hline & & $n$ & $\%$ (mean) & SD & $n$ & $\%$ (mean) & SD & \\
\hline Age (months) & 776 & 397 & (103.6) & 43.5 & 379 & (98.2) & 45.1 & 0.097 \\
\hline \multicolumn{9}{|l|}{ Sex } \\
\hline Male & 372 & 199 & 50.3 & & 173 & 45.7 & & 0.199 \\
\hline Female & 403 & 197 & 49.7 & & 206 & 54.3 & & \\
\hline \multicolumn{9}{|l|}{ Orphan-hood } \\
\hline Both parents alive & 280 & 154 & 40.6 & & 126 & 35.5 & & 0.388 \\
\hline Only mother alive & 166 & 82 & 21.6 & & 84 & 23.7 & & \\
\hline Only father alive & 117 & 54 & 14.3 & & 63 & 17.7 & & \\
\hline Both parents dead & 171 & 89 & 23.5 & & 82 & 23.1 & & \\
\hline \multicolumn{9}{|l|}{ HIV-clinical stage ${ }^{b}$} \\
\hline Stage I & 52 & 23 & 5.9 & & 29 & 8.0 & & 0.054 \\
\hline Stage ॥ & 155 & 83 & 21.3 & & 72 & 19.8 & & \\
\hline Stage III & 463 & 230 & 59.1 & & 233 & 64.2 & & \\
\hline Stage IV & 82 & 53 & 13.7 & & 29 & 8.0 & & \\
\hline \multicolumn{9}{|l|}{ On $A R T^{b}$} \\
\hline No & 96 & 54 & 13.6 & & 42 & 11.1 & & 0.293 \\
\hline Yes & 679 & 343 & 86.4 & & 336 & 88.9 & & \\
\hline \multicolumn{9}{|l|}{ ART duration ${ }^{a}$} \\
\hline Mean months & 677 & 357 & $(36.9)$ & 27.8 & 320 & $(33.5)$ & 27.5 & 0.108 \\
\hline \multicolumn{9}{|l|}{ Caregiver $^{b}$} \\
\hline Mother & 352 & 179 & 45.1 & & 173 & 45.7 & & 0.876 \\
\hline Other & 424 & 218 & 54.9 & & 206 & 54.3 & & \\
\hline \multicolumn{9}{|c|}{ Education level (caregiver) ${ }^{\mathrm{b}}$} \\
\hline Not formal & 208 & 113 & 28.5 & & 95 & 25.1 & & 0.074 \\
\hline Primary & 497 & 241 & 60.7 & & 256 & 67.7 & & \\
\hline Secondary \&above & 70 & 43 & 10.8 & & 27 & 7.2 & & \\
\hline \multicolumn{9}{|l|}{ Wealth index ${ }^{\mathrm{b}}$} \\
\hline Lowest & 159 & 108 & 27.2 & & 51 & 13.5 & & $<0.001$ \\
\hline Low & 152 & 55 & 13.9 & & 97 & 25.6 & & \\
\hline Middle & 156 & 61 & 15.4 & & 95 & 25.1 & & \\
\hline High & 154 & 76 & 19.1 & & 78 & 20.5 & & \\
\hline Highest & 155 & 97 & 24.4 & & 58 & 15.3 & & \\
\hline \multicolumn{9}{|l|}{ Food security (HFIAS) ${ }^{b}$} \\
\hline Food-secure & 230 & 126 & 31.7 & & 104 & 27.4 & & 0.190 \\
\hline Food-insecure & 546 & 271 & 68.3 & & 275 & 72.6 & & \\
\hline
\end{tabular}

${ }^{\mathrm{a}}$-test; ${ }^{\mathrm{b}} \mathrm{Chi}$-square test

pre-training test session. For example, the mean value for MLPs' knowledge score on pediatric HIV/AIDS improved from 9.8 to $14.5(p<0.001)$; knowledge on food preparation hygiene improved from 2.9 to 4.6 ( $p$ $<0.001)$; knowledge on feeding practices improved from 4.4 to $9.3(p<0.001)$; and knowledge on nutrition and feeding counseling improved from 6.4 to 8.8 $(p<0.001)$ after the training.

\section{Changes in feeding practices}

Table 3 shows the changes in feeding practices following the intervention and at final follow-up. HIV-positive children in the intervention arm had a slightly higher mean feeding frequency at baseline compared to those of the control arm (2.8 vs. 2.6, $p=0.041)$. However, a significant increase in feeding frequency was observed in the intervention arm compared to the control arm at the 
Table 2 MLPs' nutritional knowledge before and after receiving nutrition training for HIV-positive children in the intervention arm

\begin{tabular}{lllll}
\hline Aspect of knowledge & $N$ & Mean & SD & $P$ \\
\hline $\begin{array}{l}\text { Total knowledge score } \\
\text { Pre-training }\end{array}$ & 16 & 23.5 & 6.5 & $<0.001$ \\
$\begin{array}{l}\text { Post-training } \\
\text { Pediatric HIV/AIDS }\end{array}$ & 16 & 37.1 & 3.1 & \\
$\quad$ Pre-training & 16 & 9.8 & 0.9 & $<0.001$ \\
$\quad$ Post-training & 16 & 14.5 & 0.2 & \\
$\begin{array}{l}\text { Food preparation hygiene } \\
\text { Pre-training }\end{array}$ & 16 & 2.9 & 1.0 & $<0.001$ \\
$\quad$ Post-training & 16 & 4.6 & 1.0 & \\
Feeding practices & & & & \\
$\quad$ Pre-training & 16 & 4.4 & 2.1 & $<0.001$ \\
$\quad$ Post-training & 16 & 9.3 & 0.9 & \\
Nutrition counseling & & & & \\
$\quad$ Pre-training & 16 & 6.4 & 1.6 & $<0.001$ \\
Post-training & 16 & 8.8 & 1.7 & \\
\hline
\end{tabular}

final follow-up (4.4 vs. 3.1, $p<0.001)$. To achieve the WHO's stipulated feeding frequency of 5 times a day, the Number Needed to Treat (NNT) to change one child's feeding frequency was 12.1 .

Similarly, dietary diversity improved significantly from 2.8 and 2.9 among the children of the intervention and control arms, respectively for baseline scores to $4.3 \mathrm{com}-$ pared to 3.4 in the intervention and control arm respectively for follow up scores.

\section{Changes in anthropometry among HIV-positive children}

Weight was not significantly different at baseline between the HIV-positive children attending CTC intervention and control arms (Table 3). However, at the final follow-up, mean weight increased significantly within the intervention arm compared to baseline, and was significantly higher compared to that of the control arm $(22.0 \mathrm{~kg}$ vs. $20.5 \mathrm{~kg}, p=0.003)$. A mean increment weight gain of $300 \mathrm{~g}$ was also observed at follow-up compared to baseline among children of the intervention arm. Changes in weight led to changes in weight-related nutritional indices (WAZ-scores, WHZ-scores, and BMIAZ-scores). The Number needed to treat (NNT) to change underweight status for one child was 3.9.

\section{Effectiveness of the intervention in improving nutrition status through changes in feeding frequency}

Table 4 shows the results of the 2-stage instrumental variable random effects regression. In the first stage regression, feeding frequency increased significantly in the intervention arm and at the 6 months final follow-up compared with the
Table 3 Changes of feeding practices, anthropometry, and nutrition status between intervention and control arms

\begin{tabular}{|c|c|c|c|c|c|c|c|c|}
\hline \multirow[t]{2}{*}{ Variable } & \multirow[t]{2}{*}{ Total } & \multicolumn{3}{|c|}{ Intervention arm } & \multicolumn{3}{|c|}{ Control arm } & \multirow[t]{2}{*}{$P$} \\
\hline & & $n$ & Mean (\%) & SD & $n$ & Mean (\%) & SD & \\
\hline \multicolumn{9}{|c|}{ Total feeding frequency $^{\mathrm{a}}$} \\
\hline Baseline & 776 & 397 & 2.8 & 0.8 & 379 & 2.6 & 0.6 & 0.041 \\
\hline Month 6 & 745 & 383 & 4.4 & 0.7 & 362 & 3.1 & 0.8 & $<0.001$ \\
\hline \multicolumn{9}{|c|}{ Feeding frequency above $5^{b}$} \\
\hline Baseline & 776 & 12 & $(3.1)$ & - & 5 & $(1.3)$ & - & 0.105 \\
\hline Month 6 & 745 & 172 & $(44.9)$ & - & 34 & $(9.4)$ & - & $<0.001$ \\
\hline \multicolumn{9}{|c|}{ Total dietary diversity score ${ }^{a}$} \\
\hline Baseline & 776 & 397 & 2.8 & 0.7 & 379 & 2.9 & 0.9 & 0.061 \\
\hline Month 6 & 745 & 383 & 4.3 & 0.8 & 362 & 3.4 & 0.7 & $<0.001$ \\
\hline \multicolumn{9}{|c|}{ Dietary diversity at least 3/day ${ }^{b}$} \\
\hline Baseline & 776 & 276 & $(69.5)$ & - & 259 & $(68.3)$ & - & 0.772 \\
\hline Month 6 & 745 & 379 & $(99.0)$ & - & 336 & $(92.8)$ & - & $<0.001$ \\
\hline \multicolumn{9}{|l|}{ Weight $(\mathrm{kg})^{\mathrm{a}}$} \\
\hline Baseline & 776 & 337 & 21.7 & 7.4 & 379 & 20.9 & 7.9 & 0.134 \\
\hline Month 6 & 745 & 383 & 22.0 & 7.1 & 362 & 20.5 & 7.4 & 0.003 \\
\hline \multicolumn{9}{|c|}{ Weight-for-age $z$-scores ${ }^{a}$} \\
\hline Baseline & 486 & 238 & -1.5 & 1.3 & 248 & -1.6 & 1.5 & 0.229 \\
\hline Month 6 & 472 & 243 & -1.1 & 1.3 & 228 & -1.9 & 1.3 & $<0.001$ \\
\hline \multicolumn{9}{|c|}{ Weight-for-height z-scores ${ }^{a}$} \\
\hline Baseline & 160 & 80 & 0.4 & 1.8 & 80 & -0.6 & 1.9 & 0.001 \\
\hline Month 6 & 141 & 72 & 1.6 & 2.3 & 69 & -0.4 & 1.3 & $<0.001$ \\
\hline \multicolumn{9}{|c|}{ BMI-for-age $z$-scores ${ }^{a}$} \\
\hline Baseline & 774 & 396 & -0.5 & 1.6 & 378 & -0.8 & 1.8 & 0.011 \\
\hline Month 6 & 745 & 383 & 0.2 & 2.1 & 362 & -0.9 & 1.6 & $<0.001$ \\
\hline \multicolumn{9}{|c|}{ Underweight (age 6-120months) } \\
\hline Baseline & 487 & 79 & $(33.2)$ & - & 105 & $(42.2)$ & - & 0.041 \\
\hline Month 6 & 471 & 55 & $(22.6)$ & - & 104 & $(45.6)$ & - & $<0.001$ \\
\hline \multicolumn{9}{|c|}{ Thinness (age 6 months- 14 years) ${ }^{b}$} \\
\hline Baseline & 776 & 59 & $(14.9)$ & - & 69 & $(18.2)$ & - & 0.210 \\
\hline Month 6 & 745 & 46 & $(12.0)$ & - & 71 & $(19.6)$ & - & 0.004 \\
\hline
\end{tabular}

${ }^{\mathrm{a}}$-test; ${ }^{\mathrm{b}} \mathrm{Chi}$-square test

Number Needed to Treat (NNT)

Feeding frequency above $5 /$ day $=12.1$; Dietary diversity at least $3 /$ day $=1.4$;

Underweight $=3.9$; Thinness $=7.0$; Stunting $=40.7$

baseline in all the three models as follows: underweightmodel: $\beta=1.15, p<0.001$ and thinness-model: $\beta=1.19, p<$ 0.001). After adjusting for important confounders and covariates, feeding frequency generally improved at the final follow-up compared to the baseline (underweight-model: $\beta$ $=0.39, p<0.001$ and thinness-model: $\beta=0.41, p<0.001)$.

In the second stage, an increase in each unit of feeding frequency was associated with a 0.15-unit decrease in the child underweight $(p<0.001)$. Caregiver's education and food insecurity were also associated with child undernutrition. 
Table 4 Effect of the intervention on nutrition status through changes in feeding frequency: Instrumental variable random effects regression

\begin{tabular}{|c|c|c|c|c|c|c|}
\hline \multirow[t]{2}{*}{ Variable } & \multicolumn{3}{|c|}{ Underweight-model } & \multicolumn{3}{|c|}{ Thinness-model } \\
\hline & $\bar{\beta}$ & $95 \% \mathrm{Cl}$ & $P$ & $\bar{\beta}$ & $95 \% \mathrm{Cl}$ & $P$ \\
\hline \multicolumn{7}{|c|}{ First stage: Changes in feeding frequency at 6 months post-intervention } \\
\hline Intervention*follow-up & 1.15 & $0.98,1.31$ & $<0.001$ & 1.19 & $1.08,1.30$ & $<0.001$ \\
\hline Intervention & 0.12 & $-0.03,0.26$ & 0.129 & 0.12 & $-0.07,0.31$ & 0.226 \\
\hline Follow-up & 0.39 & $0.27,0.51$ & $<0.001$ & 0.41 & $0.33,0.49$ & $<0.001$ \\
\hline Age & -0.01 & $-0.01,0.01$ & 0.849 & -0.01 & $-0.01,0.01$ & 0.225 \\
\hline Sex & -0.06 & $-0.18,0.06$ & 0.333 & 0.11 & $-0.02,0.25$ & 0.111 \\
\hline Caregiver's education & 0.02 & $-0.08,0.12$ & 0.671 & -0.01 & $-0.09,0.08$ & 0.918 \\
\hline Wealth index & 0.04 & $-0.01,0.09$ & 0.100 & 0.01 & $-0.01,0.08$ & 0.079 \\
\hline Food insecurity & 0.01 & $-0.01,0.01$ & 0.892 & -0.03 & $-0.01,0.01$ & 0.380 \\
\hline \multicolumn{7}{|c|}{ Second stage: random effects regression: changes in nutrition status as a result of changes in feeding frequency } \\
\hline Feeding frequency & -0.15 & $-0.24,-0.07$ & $<0.001$ & -0.04 & $-0.08,0.01$ & 0.059 \\
\hline Intervention & -0.07 & $-0.16,0.02$ & 0.133 & -0.03 & $-0.11,0.05$ & 0.402 \\
\hline Follow-up & 0.12 & $0.03,0.21$ & 0.012 & 0.01 & $-0.03,0.06$ & 0.541 \\
\hline Age & 0.01 & $0.01,0.01$ & 0.018 & 0.01 & $0.01,0.01$ & $<0.001$ \\
\hline Sex & -0.02 & $-0.09,0.05$ & 0.575 & -0.01 & $-0.06,0.05$ & 0.832 \\
\hline Caregiver's education & -0.01 & $-0.06,0.05$ & 0.917 & -0.02 & $-0.06,0.01$ & 0.204 \\
\hline Wealth index & -0.02 & $-0.05,0.01$ & 0.083 & -0.01 & $-0.02,0.01$ & 0.691 \\
\hline Food insecurity & 0.01 & $-0.01,0.01$ & 0.514 & -0.01 & $-0.01,0.01$ & 0.154 \\
\hline
\end{tabular}

Intervention*follow-up = interaction term between intervention and follow-up

Intervention: subjects at the intervention compared to control arm

Follow-up: subjects at the follow-up compared to the baseline

\section{Effectiveness of the intervention in improving nutrition status through changes in dietary diversity}

After adjusting for important confounders and covariates, feeding frequency generally improved at the final followup compared to the baseline (underweight-model: $\beta=$ 0.48, $p<0.001$ and thinness-model: $\beta=0.46, p<0.001$ ) (Table 5). In the second stage, an increase in one unit of dietary diversity was associated with a 0.16-unit decrease in the child underweight $(p<0.001)$ but not thinness $(p=$ 0.078). Other factors associated with undernutrition included age, wealth index and food insecurity.

\section{Discussion}

This is the first cluster-randomized controlled trial to examine the efficacy of nutrition training in improving MLPs' nutrition education. It also serves as the first study to examine the efficacy of such training using standard WHO guidelines [10] and local determinants of undernutrition [6] toward improving feeding practices and nutrition status among HIV-positive children. In this study, nutrition training of MLPs improved their nutrition knowledge. It also improved feeding frequency and dietary diversity among HIV-positive children at the 6-month follow-up in the intervention arm. As a result, a small but significant weight gain and related improvements in nutrition statuses were detected among HIV-positive children of the intervention arm.

The following three pathways may help explain such gains. First, the nutrition training improved the nutrition knowledge of MLPs in the intervention arm. In Tanga, MLPs who care for HIV-positive children, had a low level of nutrition knowledge before the training [6]. However, through this intervention, they could improve their knowledge significantly and thus exert positive influences on the caregivers as explained below.

Second, the feeding practices improved significantly among HIV-positive children in the intervention arm. In the formative research [6], 88.1\% of 748 children had a feeding frequency lower than that recommended by the WHO for HIV-positive children [10]. About $62 \%$ of them also had low levels of dietary diversity. These factors were positively associated with poor nutrition statuses [6]. The mean feeding frequency and dietary diversity increased more in the intervention than the control arm. Even after adjusting for other variables, the intervention arm at follow-up had a significantly higher feeding frequency and dietary diversity. Therefore, nutrition training coupled with nutrition counseling improved feeding practices to a level similar to that observed in general and HIV-negative populations [13]. In this study, the trained MLPs could 
Table 5 Effect of the intervention on nutrition status through changes in dietary diversity: Instrumental variable random effect regression

\begin{tabular}{|c|c|c|c|c|c|c|}
\hline \multirow[t]{2}{*}{ Variable } & \multicolumn{3}{|c|}{ Underweight-model } & \multicolumn{3}{|c|}{ Thinness-model } \\
\hline & $\beta$ & $95 \% \mathrm{Cl}$ & P & $\beta$ & $95 \% \mathrm{Cl}$ & $P$ \\
\hline Intervention*follow-up & 1.11 & $0.94,1.28$ & $<0.001$ & 1.10 & $0.96,1.24$ & $<0.001$ \\
\hline Intervention & -0.08 & $-0.22,0.07$ & 0.310 & -0.07 & $-0.19,0.05$ & 0.254 \\
\hline Follow-up & 0.48 & $0.35,0.60$ & $<0.001$ & 0.46 & $0.36,0.56$ & $<0.001$ \\
\hline Age & 0.01 & $-0.01,0.01$ & 0.911 & -0.01 & $-0.01,0.01$ & 0.349 \\
\hline Sex & -0.06 & $-0.17,0.06$ & 0.354 & 0.01 & $-0.10,0.10$ & 0.973 \\
\hline Caregiver's education & 0.03 & $-0.07,0.13$ & 0.544 & 0.02 & $-0.06,0.10$ & 0.629 \\
\hline Wealth index & 0.01 & $-0.04,0.06$ & 0.583 & 0.01 & $-0.03,0.04$ & 0.865 \\
\hline Food insecurity & -0.01 & $-0.02,-0.01$ & 0.001 & -0.01 & $-0.02,-0.01$ & $<0.001$ \\
\hline \multicolumn{7}{|c|}{ Second stage: random effects regression: changes in nutrition status as a result of changes in dietary diversity } \\
\hline \multirow[t]{2}{*}{ Variable } & \multicolumn{3}{|c|}{ Underweight (WAZ <-2SD) } & \multicolumn{3}{|c|}{ Thinness (BMIAZ <-2SD) } \\
\hline & $\beta$ & $95 \% \mathrm{Cl}$ & $P$ & $\beta$ & $95 \% \mathrm{Cl}$ & $P$ \\
\hline Dietary diversity & -0.16 & $-0.25,-0.07$ & 0.001 & -0.05 & $-0.10,0.01$ & 0.078 \\
\hline Intervention & -0.10 & $-0.18,-0.01$ & 0.022 & -0.04 & $-0.09,0.01$ & 0.121 \\
\hline Follow-up & 0.13 & $0.03,0.24$ & 0.015 & 0.03 & $-0.04,0.09$ & 0.408 \\
\hline Age & 0.01 & $0.01,0.01$ & 0.021 & 0.01 & $0.01,0.01$ & $<0.001$ \\
\hline Sex & -0.02 & $-0.09,0.05$ & 0.614 & -0.01 & $-0.05,0.04$ & 0.887 \\
\hline Caregiver's education & 0.01 & $-0.06,0.21$ & 0.934 & -0.03 & $-0.07,0.01$ & 0.073 \\
\hline Wealth index & -0.03 & $-0.06,0.06$ & 0.047 & -0.01 & $-0.03,0.01$ & 0.108 \\
\hline Food insecurity & -0.01 & $-0.01,0.01$ & 0.684 & -0.01 & $-0.01,-0.01$ & 0.022 \\
\hline
\end{tabular}

Intervention*follow-up = interaction term between intervention and follow-up Intervention: subjects at the intervention compared to control arm

Follow-up: subjects at the follow-up compared to the baseline

thus help to transmit nutrition knowledge to caregivers $[11,15,35]$, and used available resources to improve feeding practices for their children $[15,36]$.

Third, the improvement of feeding practices brought a modest weight gain among the observed HIV-positive children in the intervention arm. After adjusting for potential differences between and within groups, improved feeding practices were associated with better nutrition statuses. Higher feeding frequency is known to increase the amount of food absorbed and replenishes losses sustained through catabolic processes triggered by HIV and opportunistic infections [10, 37-39]. Increased dietary diversity also improves appetite and thereby increases the amount of food consumed by a child, even apart from the added nutritional value $[10,39]$. The concomitant increases in both factors must have contributed to the observed weight gain among children in the intervention arm.

Despite using a randomized controlled design, our study was not free of limitations. First, we depended on selfreport and the recall of caregivers in measuring feeding practices. However, the current findings at baseline were not significantly different from those identified in the formative research [6]. Second, we lost a total of 31 pairs of HIV-positive children and their caregivers in our final analyses, as we could not interview children who came alone to the CTCs unaccompanied by their caregivers [25]. However, such children were almost evenly distributed in both arms. Third, a 6-month follow-up may be too short to observe significant changes in long-term outcome variables such stunting. Therefore, we could not see the impact of this intervention in stunting that need a relatively longer follow-up time, an avenue for future studies. Fourth, some questionnaire had missing data on some variables. This led into small differences in total values in some demographic characteristics. We did not exclude questionnaires with missing variables unless they also had missing information on outcome variables. Fifth, we cannot ascertain any changes in the nutrition knowledge for caregiver, as we did not measure it before, during, and after the intervention. This is an important area for future research. Despite its limitations, this is the first study to assess the efficacy of nutrition training toward improving MLPs' feeding practices and, thereby, the nutrition status of HIV-positive children.

\section{Conclusions}

In conclusion, this study found out that, providing nutrition training to MLPs effectively improved their nutrition knowledge, which in turn improved feeding practices 
among HIV-positive children in Tanga region, Tanzania. The improved feeding practices brought about a small weight gain in this food-secure region. Even where the health workforce is limited, providing nutrition training to the available workforce can help to ameliorate undernutrition among HIV-positive children. Nutrition training alone, however, may not be enough to ameliorate growth faltering. Efforts are thus needed to improve food insecurity, poverty, and education levels among the caregivers of HIV-positive children toward bringing about lasting and sustainable improvements in nutrition.

\section{Abbreviations}

ART: Antiretroviral therapy; BMIAZ: Body Mass Index-for-Age Z-score; Cl: Confidence interval; CTC: Care and treatment center; CTC: Care and treatment center; HIV: Human immunodeficiency virus; MLP: Mid level provider; NNT: Number Needed to Treat; OR: Odds ratio; PEER: Patient expected event rate; SD: Standard Deviation; UK: United Kingdoms; USA: United States of America; WAZ: Weight-for-Age Z-score; WHO: World Health Organization; WHZ: Weight-for-Height Z-score.

\section{Acknowledgments}

The authors would like to thank Dr. Adelheid Onyango and Dr. Maria Del Carmen Casanovas (World Health Organization) for providing us with the nutrition training materials and their invaluable inputs toward the conduct of this trial. We also thank Dr. John Lusingu and his team (NIMR Korogwe) for providing us the venue for the training. Dr. Selemani Msangi (RACC), Dr. Uredi Ally (RMO), Dr. Robert Kissanga (AIDS Relief), and all DMOs and all CTC in-charges who gave permission for their staff to attend this training.

\section{Funding}

This study was funded by a grant from the Directorate of Research and Publication of the Muhimbili University of Health and Allied Sciences through SIDA-SAREC grant (Ref. No: MU/01/1022/0130/21), and the Ministry of Health, Labor and Welfare of Japan (Research Grant No: H24-Chikyukibo-Ippan-008). Funders did not have any influence on the results or conduct of this study.

\section{Availability of data and materials}

Dataset used during the current study are available from the corresponding author on reasonable request.

\section{Authors' contributions}

BFS conceived the research questions, designed the study, conducted the nutrition training and data collection and analyses, and prepared the first draft. DPU contributed in protocol development, involved in drafting the manuscript, and revise the manuscript critically. LBM critically revised the manuscript. KCP refined the research questions, revised the protocol, and revised the manuscript. NPM and OSU conducted both theoretical and practical parts of the nutritional training and revised the manuscript. GHL and ATK were involved in protocol development, spearheaded the IRB process, and revised the manuscript. AS conducted analyses and revised the manuscript. JY and KN participated in the preparation of the protocol development and the first draft of the manuscript. MJ reviewed the study protocol and manuscript, supervised training and data collection, and approved the submission. All authors read and approved the final version of the manuscript for submission.

\section{Competing interests}

The authors declare that they have no competing interests.

\section{Consent for publication}

Not applicable.

\section{Ethics approval and consent to participate}

This study was approved by the Research Ethics Committee of the University of Tokyo (reference number: 1007-(1), and the Expedited Review Sub-committee of the Senate Research and Publication Directorate of the Muhimbili University of Health and Allied Sciences reference number: MU/DRP/AECNoI.XVI/88. Caregivers of children below 18 years of age provided the written informed consent after the study was explained to them for their children to participate in this study. All adult participants provided the written informed consent before participate. This included MLPs who received the nutrition training. The protocol was registered on 15/02/2013, before the recruitment at ISRCTN trial registry with the trial registration number: ISRCTN65346364.

\section{Publisher's Note}

Springer Nature remains neutral with regard to jurisdictional claims in published maps and institutional affiliations.

\section{Author details}

'School of Public Health and Social Sciences, Muhimbili University of Health and Allied Sciences, P.O Box 65489, Dar es Salaam, Tanzania. ${ }^{2}$ Department of Community and Global Health, Graduate School of Medicine, The University of Tokyo, 7-3-1, Hongo, Bunkyo-ku, Tokyo 113-0033, Japan. ${ }^{3}$ Department of Public Health, School of Public Health and Health Sciences, University of Massachusetts Amherst, Arnold House, 715 North Pleasant St, Amherst, MA 01003-9304, USA. ${ }^{4}$ Muhimbili National Hospital, P.O. Box 65000, Dar es Salaam, Tanzania.

Received: 14 October 2015 Accepted: 18 March 2017

Published online: 04 April 2017

\section{References}

1. Sunguya BF, Ong KI, Dhakal S, Mlunde LB, Shibanuma A, Yasuoka J, Jimba M. Strong nutrition governance is a key to addressing nutrition transition in low and middle-income countries: review of countries' nutrition policies. Nutr J. 2014;13(1):65.

2. UNICEF. Improving child nutrition: the achievable imparatives for global progress. In. New York: United Nations Children's Fund; 2013.

3. Lutter CK, Daelmans BM, de Onis M, Kothari MT, Ruel MT, Arimond M, Deitchler M, Dewey KG, Blössner M, Borghi E. Undernutrition, poor feeding practices, and low coverage of key nutrition interventions. Pediatrics. 2011; 128(6):e1418-27.

4. Kadiyala S, Rawat R. Food access and diet quality independently predict nutritional status among people living with HIV in Uganda. Public Health Nutr. 2013;16(1):164-70.

5. Sunguya BF, Poudel KC, Otsuka K, Yasuoka J, Mlunde LB, Urassa DP, Mkopi NP, Jimba M. Undernutrition among HIV-positive children in Dar es Salaam, Tanzania: antiretroviral therapy alone is not enough. BMC Public Health. 2011;11:869.

6. Sunguya BF, Poudel KC, Mlunde LB, Urassa DP, Yasuoka J, Jimba M. Poor Nutrition Status and Associated Feeding Practices among HIV-Positive Children in a Food Secure Region in Tanzania: A Call for Tailored Nutrition Training. PLoS One. 2014;9(5):e98308.

7. Ivers LC, Cullen KA, Freedberg KA, Block S, Coates J, Webb P. HIV/AIDS, undernutrition, and food insecurity. Clin Infect Dis. 2009;49(7):1096-102.

8. Ivers LC, Cullen KA. Food insecurity: special considerations for women. Am J Clin Nutr. 2011;94(6):1740S-4S.

9. Weiser SD, Young SL, Cohen CR, Kushel MB, Tsai AC, Tien PC, Hatcher AM, Frongillo EA, Bangsberg DR. Conceptual framework for understanding the bidirectional links between food insecurity and HIV/AIDS. Am J Clin Nutr. 2011;94(6):1729S-39S.

10. WHO. Guidelines for an integrated approach to the nutritional care of HIV-infected children (6 months to 14 years). Geneva: World Health Organization; 2009.

11. Sunguya BF, Poudel KC, Mlunde LB, Urassa DP, Yasuoka J, Jimba M. Nutrition Training Improves Health Workers' Nutrition Knowledge and Competence to Manage Child Undernutrition: A Systematic Review. Front Public Health. 2013;1:37.

12. Pelto GH, Santos I, Gonçalves H, Victora C, Martines J, Habicht JP. Nutrition counseling training changes physician behavior and improves caregiver knowledge acquisition. J Nutr. 2004;134(2):357-62.

13. Sunguya BF, Poudel KC, Mlunde LB, Shakya P, Urassa DP, Jimba M, Yasuoka J. Effectiveness of nutrition training of health workers toward improving caregivers' feeding practices for children aged six months to two years: a systematic review. Nutr J. 2013;12:66.

14. Imdad A, Yakoob MY, Bhutta ZA. Impact of maternal education about complementary feeding and provision of complementary foods on child growth in developing countries. BMC Public Health. 2011;11 Suppl 3:S25. 
15. Dewey KG, Adu-Afarwuah S. Systematic review of the efficacy and effectiveness of complementary feeding interventions in developing countries. Matern Child Nutr. 2008;4 Suppl 1:24-85.

16. Bhandari N, Mazumder S, Bahl R, Martines J, Black RE, Bhan MK, Group IFS. An educational intervention to promote appropriate complementary feeding practices and physical growth in infants and young children in rural Haryana, India. J Nutr. 2004;134(9):2342-8.

17. Santos I, Victora CG, Martines J, Gonçalves H, Gigante DP, Valle NJ, Pelto G. Nutrition counseling increases weight gain among Brazilian children. J Nutr. 2001;131(11):2866-73.

18. Bhandari N, Bahl R, Nayyar B, Khokhar P, Rohde JE, Bhan MK. Food supplementation with encouragement to feed it to infants from 4 to 12 months of age has a small impact on weight gain. J Nutr. 2001;131(7):1946-51.

19. Roy SK, Fuchs GJ, Mahmud Z, Ara G, Islam S, Shafique S, Akter SS, Chakraborty B. Intensive nutrition education with or without supplementary feeding improves the nutritional status of moderately-malnourished children in Bangladesh. J Health Popul Nutr. 2005:23(4):320-30.

20. Zaman S, Ashraf RN, Martines J. Training in complementary feeding counselling of healthcare workers and its influence on maternal behaviours and child growth: a cluster-randomized controlled trial in Lahore, Pakistan. J Health Popul Nutr. 2008;26(2):210-22.

21. Vazir S, Engle P, Balakrishna N, Griffiths PL, Johnson SL, Creed-Kanashiro H, Fernandez Rao S, Shroff MR, Bentley ME. Cluster-randomized trial on complementary and responsive feeding education to caregivers found improved dietary intake, growth and development among rural Indian toddlers. Matern Child Nutr. 2013;9(1):99-117.

22. Pachón H, Schroeder DG, Marsh DR, Dearden KA, Ha TT, Lang TT. Effect of an integrated child nutrition intervention on the complementary food intake of young children in rural north Viet Nam. Food Nutr Bull. 2002;23(4 Suppl):62-9.

23. GHWA. Mid-level health providers a promising resource to achieve the health Millennium Development Goals. Geneva: World Health Organization; 2010.

24. Global Atlas of the Health Workforce. http://apps.who.int/globalatlas/default.asp.

25. Sunguya BF, Poudel KC, Mlunde LB, Urassa DP, Jimba M, Yasuoka J. Efficacy of in-service nutrition training for mid-level providers to improve feeding practices among HIV-positive children in Tanga, Tanzania: study protocol for a cluster randomized controlled trial. Trials. 2013;14(1):352.

26. NBS, ORC-Macro. Tanzania Demographic and Health Survey 2009-2010. Dar es Salaam: National Bureau of Statistics and ORC Macro; 2010.

27. Sunguya BF, Poudel KC, Mlunde LB, Otsuka K, Yasuoka J, Urassa DP, Mkopi NP, Jimba M. Ready to Use Therapeutic Foods (RUTF) improves undernutrition among ART-treated, HIV-positive children in Dar es Salaam, Tanzania. Nutr J. 2012;11:60

28. de Onis M, Onyango AW, Van den Broeck J, Chumlea WC, Martorell R. Measurement and standardization protocols for anthropometry used in the construction of a new international growth reference. Food Nutr Bull. 2004; 25(1 Suppl):S27-36.

29. Roche AF, Davila GH. Differences between recumbent length and stature within individuals. Growth. 1974;38(3):313-20.

30. WHO. Multicenter Growth Reference Study Group. WHO Child Growth Standards based on length, weight and age. Acta Paediatrica. 2006:450:76-84

31. WHO. Multicentre Growth Reference Study Group. WHO Child Growth Standards: Growth velocity based on weight, length and head circumference: Methods and development. Geneva: World Health Organization; 2009.

32. WHO. WHO AnthroPlus for personal computers Manual: Software for assessing growth of the world's children and adolescents. Geneva: World Health Organization; 2009.

33. Martens EP, Pestman WR, de Boer A, Belitser SV, Klungel OH. Instrumental variables: application and limitations. Epidemiology. 2006;17(3):260-7.

34. Albers L, Heinen F, Landgraf M, Straube A, Blum B, Filippopulos F, Lehmann S, Mansmann U, Berger U, Akboga Y, et al. Headache cessation by an educational intervention in grammar schools: a cluster randomized trial. Eur J Neurol. 2015; 22(2):270-6.

35. Shi L, Zhang J. Recent Evidence of the Effectiveness of Educational Interventions for Improving Complementary Feeding Practices in Developing Countries. J Trop Pediatr. 2011;57(2):91-8.

36. Shi L, Zhang J, Wang Y, Caulfield LE, Guyer B. Effectiveness of an educational intervention on complementary feeding practices and growth in rural China: a cluster randomised controlled trial. Public Health Nutr. 2010;13(4):556-65.
37. Piwoz E, Preble E. HIV/AIDS and nutrition: a review of literature and recommendations for nutritional care and support in Sub-Saharan Africa. Washington, D.C: Academy for Educational Development; 2000.

38. Guarino A, Bruzzese E, De Marco G, Buccigrossi V. Management of gastrointestinal disorders in children with HIV infection. Paediatr Drugs. 2004;6(6):347-62.

39. WHO. Nutrient requirements for people living with HIV/AIDS: report of a technical consultation. Geneva: World Health Organization; 2003.

\section{Submit your next manuscript to BioMed Central and we will help you at every step:}

- We accept pre-submission inquiries

- Our selector tool helps you to find the most relevant journal

- We provide round the clock customer support

- Convenient online submission

- Thorough peer review

- Inclusion in PubMed and all major indexing services

- Maximum visibility for your research

Submit your manuscript at www.biomedcentral.com/submit
) Biomed Central 